\title{
DNA barcoding reveals overlooked shark and bony fish species in landing reports of small-scale fisheries from northern Peru
}

Alan Marín, Renato Gozzer Wuest, Jorge Grillo, Irina B. Alvarez-Jaque, Juan Carlos Riveros. 2022. Marine and Fishery Sciences 35(2). DOI: https://doi.org/10.47193/mafis.3522022010502

Table S1. Latitude and longitude for all fish landing sites of SSF from northern Peru used in this study to obtain fin clips for DNA identification analyses

\begin{tabular}{|c|c|c|}
\hline Region & Fish landing site & Latitude and Longitude \\
\hline \multirow[t]{8}{*}{ Piura } & Bayóvar & S 549'20.64" W 81ํ'56.28" \\
\hline & Cabo Blanco & 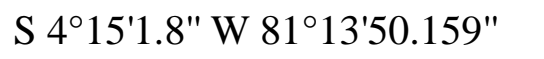 \\
\hline & El Nuro & $\mathrm{S} 4^{\circ} 13^{\prime} 11.424^{\prime \prime} \mathrm{W} 81^{\circ} 10^{\prime} 52.32^{\prime \prime}$ \\
\hline & La Islilla & S 5¹2'39.24" W 81¹1'39.84" \\
\hline & La Tortuga & S 516'49.44" W 817'22.079" \\
\hline & Los Órganos & S 410'42.24" W 818'3.12" \\
\hline & Paita & S 5 5'1.32" W 81ํ'ㄱ" \\
\hline & Yacila & S 57'51.6" W 81¹0'17.399" \\
\hline \multirow[t]{4}{*}{ Tumbes } & Caleta Grau & S 339'52.2" W 80³7'57.359" \\
\hline & Cancas & S 356'42.36" W 8056'22.92" \\
\hline & La Cruz & S 338'1.68" W 80³5'13.919" \\
\hline & Puerto Pizarro & S 329'59.64" W 80²3'25.8" \\
\hline
\end{tabular}

Table S2. List of primers used in DNA barcoding for genetic species identification of specimens from SSF in northern Peru

\begin{tabular}{lllll}
\hline $\begin{array}{l}\text { Primer } \\
\text { name }\end{array}$ & Direction & Sequence (5'-3') & $\begin{array}{l}\text { Target } \\
\text { gene/region }\end{array}$ & Reference \\
\hline FishF1 & Forward & TCAACCAACCACAAAGACATTGGCAC & COI & Ward et al. (2005) \\
FishR1 & Reverse & TAGACTTCTGGGTGGCCAAAGAATCA & & \\
A & Forward & TTCCACCTCTAACTCCCAAAGCTAG & D-loop & Lee et al. (1995) \\
G & Reverse & CGTCGGATCCCATCTTCAGTGTTATGCTT & & \\
\hline
\end{tabular}




\section{Supplementary Material}

Table S3. List of totalspecies identified through DNA barcoding

\begin{tabular}{|c|c|c|c|c|c|c|c|}
\hline Family & $\begin{array}{l}\text { Species Match ID } \\
\text { (BOLD/NCBI) }\end{array}$ & Overlooked & Label & Sampling site & $\mathbf{n}$ & GenBank access & IUCN \\
\hline \multirow{6}{*}{ Carangidae } & Seriola rivoliana & NO & Fortuno & Cancas, Tumbes & 1 & MN880603 & $\mathrm{LC}$ \\
\hline & \multirow{5}{*}{ Trachinotus paitensis } & \multirow{5}{*}{ NO } & \multirow{5}{*}{ Pampano } & \multirow{5}{*}{ La Cruz, Tumbes } & \multirow{5}{*}{5} & MN880583 & \\
\hline & & & & & & MN880584 & \\
\hline & & & & & & MN880585 & $\mathrm{LC}$ \\
\hline & & & & & & MN880586 & \\
\hline & & & & & & MN880587 & \\
\hline \multirow[t]{2}{*}{ Centrolophidae } & $\begin{array}{l}\text { Schedophilus } \\
\text { haedrichi }\end{array}$ & NO & Mocosa & Cabo Blanco, Piura & 1 & MN880515 & $\mathrm{LC}$ \\
\hline & Seriolella violacea & NO & Cojinoba & Bayóvar, Piura & 1 & MN880601 & $\mathrm{LC}$ \\
\hline Centropomidae & $\begin{array}{l}\text { Centropomus } \\
\text { unionensis }\end{array}$ & YES & Robalo plateado & Cancas, Tumbes & 1 & MN880528 & $\mathrm{LC}$ \\
\hline \multirow{2}{*}{ Haemulidae } & $\begin{array}{l}\text { Anisotremus } \\
\text { interruptus }\end{array}$ & NO & Berrugata & La Tortuga, Piura & 1 & MN880558 & $\mathrm{LC}$ \\
\hline & $\begin{array}{l}\text { Anisotremus } \\
\text { scapularis }\end{array}$ & NO & Chita & La Islilla, Piura & 1 & MN880542 & $\mathrm{LC}$ \\
\hline Labridae & Decodon melasma & YES & San Pedrano & La Islilla, Piura & 1 & MN880543 & $\mathrm{LC}$ \\
\hline Lutjanidae & $\begin{array}{l}\text { Lutjanus } \\
\text { argentiventris }\end{array}$ & YES & $\begin{array}{l}\text { Paramo rojo } \\
\text { Paramo muelon }\end{array}$ & Cancas, Tumbes & 2 & $\begin{array}{l}\text { MN880602 } \\
\text { MN880525 }\end{array}$ & $\mathrm{LC}$ \\
\hline
\end{tabular}




\section{Supplementary Material}

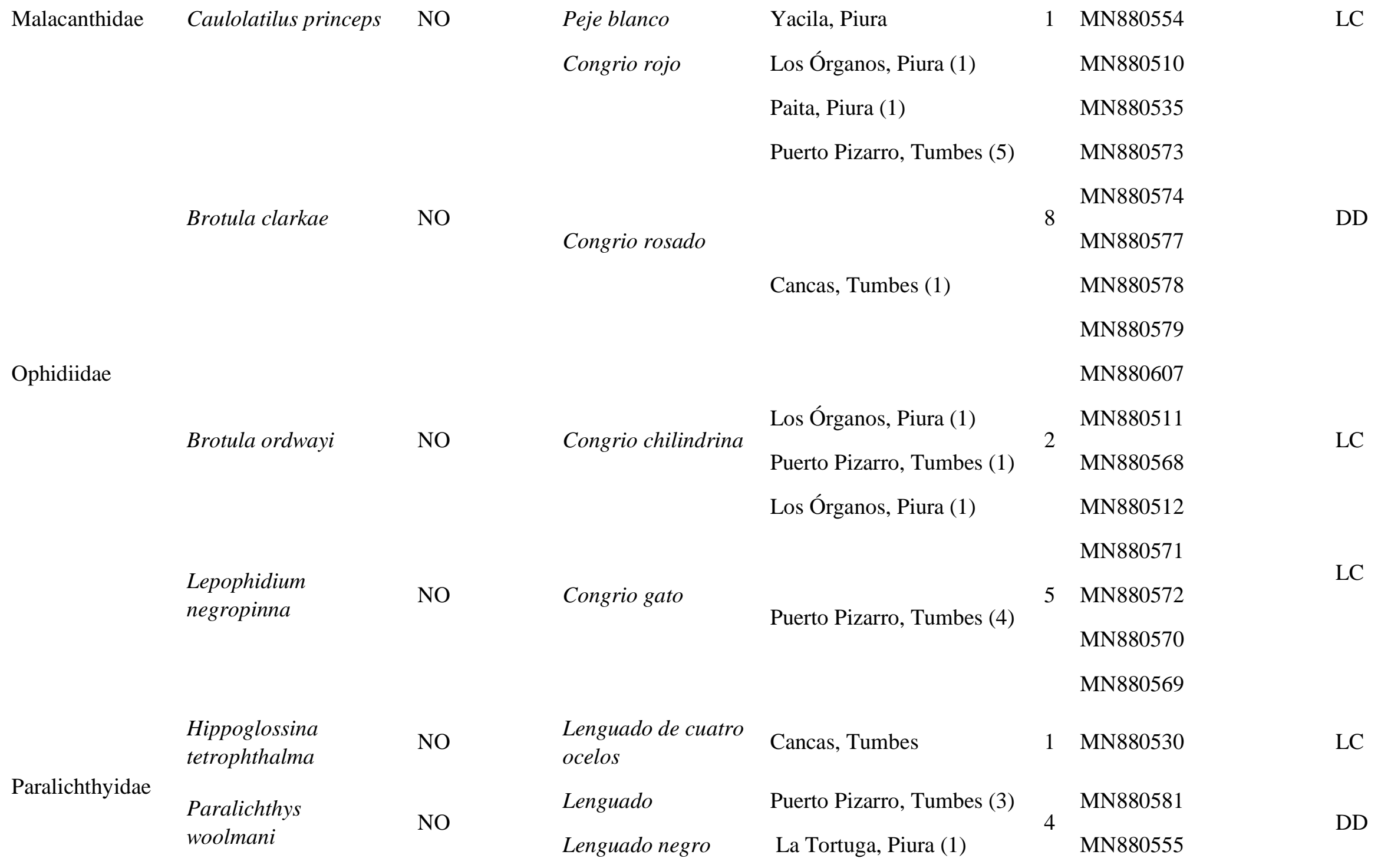




\section{Supplementary Material}

\begin{tabular}{|c|c|c|c|c|c|c|}
\hline & & & & & & $\begin{array}{l}\text { MN880580 } \\
\text { MN880582 }\end{array}$ \\
\hline & Cynoscion analis & NO & Cachema & Cabo Blanco, Piura & 1 & MN880521 \\
\hline & $\begin{array}{l}\text { Cynoscion } \\
\text { phoxocephalus }\end{array}$ & NO & Ayanque/Cachema & Cancas, Tumbes & 1 & MN880604 \\
\hline Sciaenidae & Cynoscion stolzmanni & NO & $\begin{array}{l}\text { Cherela cola } \\
\text { amarilla }\end{array}$ & Cancas, Tumbes & 1 & MN880529 \\
\hline & Pareques lanfeari & NO & Camiseta & Cancas, Tumbes & 1 & MN880527 \\
\hline & Larimus acclivis & YES & Bereche & La Tortuga, Piura & 1 & MN880556 \\
\hline & Umbrina xanti & NO & Polla & Caleta Grau, Tumbes & 1 & MN880599 \\
\hline & Scomberomorus sierra & NO & Sierra & Cabo Blanco, Piura & 1 & MN880519 \\
\hline Scombridae & Thunnus albacares & NO & $\begin{array}{l}\text { Atún } \\
\text { Atún ojo grande }\end{array}$ & $\begin{array}{l}\text { El Nuro, Piura (1) } \\
\text { Cabo Blanco, Piura (1) }\end{array}$ & 2 & $\begin{array}{l}\text { MN880522/MN880618 } \\
\text { MN880518/MN880616 }\end{array}$ \\
\hline & Acanthistius pictus & NO & Cherlo & La Islilla, Piura & 1 & MN880541 \\
\hline & Alphestes immaculatus & NO & Mero pintado & Cancas, Tumbes & 1 & MN880524 \\
\hline & Diplectrum pacificum & NO & Carajito & Puerto Pizarro, Tumbes & 1 & MN880597 \\
\hline Serranidae & Epinephelus analogus & NO & Mero Pintado & Puerto Pizarro, Tumbes & 1 & MN880560 \\
\hline & $\begin{array}{l}\text { Epinephelus } \\
\text { quinquefasciatus }\end{array}$ & NO & Mero ojo chico & Puerto Pizarro, Tumbes & 1 & MN880588 \\
\hline & Hemanthias peruanus & NO & Carapachudo & La Cruz, Tumbes & 1 & MN880600 \\
\hline
\end{tabular}




\section{Supplementary Material}

\begin{tabular}{|c|c|c|c|c|c|}
\hline Hemilutjanus & NO & Sis do & Cabo Blanco, Piura (1) & 2 & MN880513 \\
\hline macrophthalmos & 10 & ojo aе ava & Puerto Pizarro, Tumbes (1) & 2 & MN880589 \\
\hline $\begin{array}{l}\text { Hyporthodus } \\
\text { acanthistius }\end{array}$ & NO & Mero rojo & Puerto Pizarro, Tumbes (1) & 1 & MN880575 \\
\hline & & Mero pescado & Los Órganos, Piura (1) & & MN880506 \\
\hline & & Mero marrón & Cabo Blanco, Piura (1) & & MN880520 \\
\hline & & Mero ojon & Paita, Piura (2) & & MN880536 \\
\hline & & & Puerto Pizarro, Tumbes (4) & & MN880537 \\
\hline $\begin{array}{l}\text { Hyporthodus } \\
\text { niphobles }\end{array}$ & NO & & & 9 & MN880566 \\
\hline & & Mero pintado & & & MN880567 \\
\hline & & & Cancas, Tumbes (1) & & MN880576 \\
\hline & & & & & MN880590 \\
\hline & & & & & MN880608 \\
\hline & & Mero murique & Los Órganos, Piura (1) & & MN880516 \\
\hline & & & La Tortuga, Piura (1) & & MN880557 \\
\hline & & & & & MN880562 \\
\hline $\begin{array}{l}\text { Mycteroperca } \\
\text { xenarcha }\end{array}$ & NO & Mero cherlo & & 7 & MN880563 \\
\hline & & & Puerto Pizarro, Tumbes (5) & & MN880564 \\
\hline & & & & & MN880565 \\
\hline & & & & & MN880561 \\
\hline
\end{tabular}

DD

VU

LC

DD 


\section{Supplementary Material}

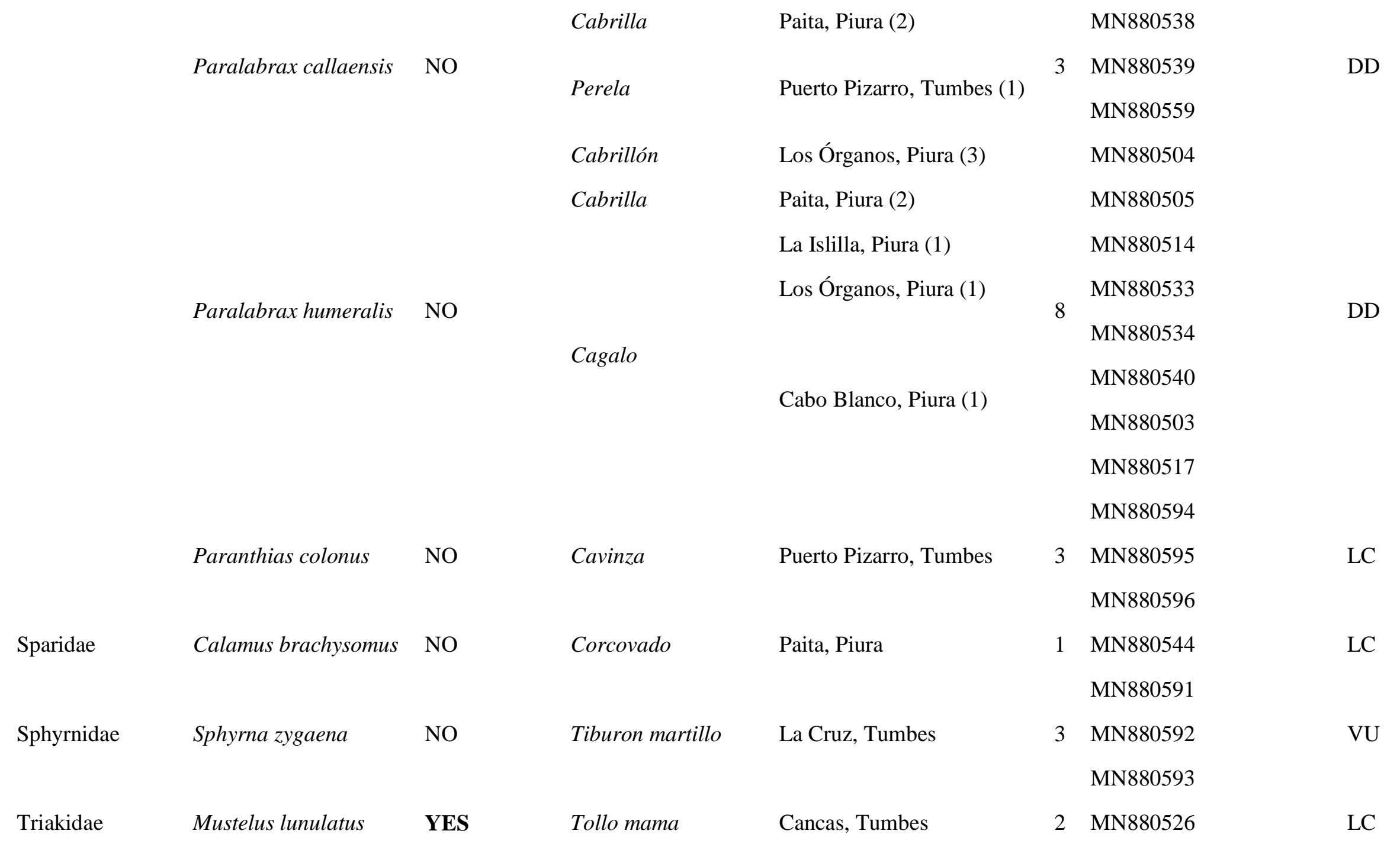




\section{Supplementary Material}

\begin{tabular}{|c|c|c|c|c|c|c|}
\hline & & & & & MN880605 & \\
\hline \multirow{6}{*}{ Mustelus whitneyi } & \multirow{6}{*}{ NO } & \multirow[t]{2}{*}{ Tollo mamita } & \multirow[t]{2}{*}{ Los Órganos, Piura (3) } & & MN880507 & \\
\hline & & & & & MN880508 & \\
\hline & & \multirow[t]{4}{*}{ Tollo mama } & \multirow[t]{4}{*}{ Cancas, Tumbes (3) } & \multirow[t]{4}{*}{6} & MN880509 & \multirow[t]{4}{*}{$\mathrm{CR}$} \\
\hline & & & & & MN880531 & \\
\hline & & & & & MN880532 & \\
\hline & & & & & MN880606 & \\
\hline
\end{tabular}

